\title{
EFFECT OF BODY POSITIONING DURING NASAL CPAP
}

\author{
M. Brunherotti, F. Martinez
}

Pediatrics, University of São Paulo, Ribeirão Preto, Brazil

Background: The effects of body positioning (BP) of preterm infants (PT) receiving nasal CPAP was not well evaluated in the literature.

Objective: To evaluate the possible effect of four BP (prone, supine, left and right lateral) on respiratory parameters of PT using nasal CPAP.

Methods: Sixteen infants with $<37$ weeks and $<2500 \mathrm{~g}$ receiving nasal CPAP were studied. Each PT was submitted to the four BP; supine (A), right lateral (B), prone (C) and left lateral (D), in one of the four randomized sequences.1- $A, D, C, B$; 2- $C, A, B, D ; 3-B, C, D$, $A$ e 4- $D, B, A$, $C$. Data were collected every 10 minutes during the 60 minutes that the infant remained at the position.

Results: Infants had GA of $29.7 \pm 2$ weeks, BW of $1353 \pm 280 \mathrm{~g}, 37.5 \%$ receive antenatal corticosteroids, 9 (56.3) were male. Infants received 5 minutes Apgar of 8.7 (6 to 10), and were 2.7 (1 to 7) days old. They were in very stable clinical condition. With mean Silverman \& Anderson bulletin (SAB) of 0.25 (0 to 1), receiving $\leq 30 \%$ of oxygen and PEEP ranging from 4 to 6 . No statistical or clinical relevant difference regarding respiratory and cardiac frequency, respiratory pause, SAB or oxygen saturation was found. Nasal prong was more frequently out of nostrils at prone position $(56,25 \%)$ than other BP $(p<0.01)$.

Conclusion: Respiratory parameters of clinically stable preterm infants receiving nasal CPAP are not affected by BP. At prone position nasal prong was found more frequently out of nostrils. 